\title{
Inner Approximation of Distance Constraints with Existential Quantification of Parameters
}

\author{
Carlos Grandón \\ Projet COPRIN, INRIA (Sophia-Antipolis) \\ Carlos.Grandon@sophia.inria.fr
}

\author{
Alexandre Goldsztejn \\ University of Nice-Sophia-Antipolis \\ Alexandre@Goldsztejn.com
}

\begin{abstract}
This paper presents and compares two methods for checking if a box is included inside the solution set of an equality constraint with existential quantification of its parameters. We focus on distance constraints, where each existentially quantified parameter has only one occurrence, because of their usefulness and their simplicity. The first method relies on a specific quantifier elimination based on geometric considerations whereas the second method relies on computations with generalized intervals (interval whose bounds are not constrained to be ordered). We show that on two dimensions problems, the two methods yield equivalent results. However, when dealing with higher dimensions, generalized intervals are more efficient.
\end{abstract}

\section{Categories and Subject Descriptors}

G.1 [Numerical Analysis]: Miscellaneous

\section{General Terms}

Theory, Algorithms

\section{Keywords}

Inner Approximation, distance constraint, AE-solution set, quantifier elimination, generalized intervals.

\section{INTRODUCTION}

There are different situations where the solution set of a constraint satisfaction problem (CSP) has a non-null volume, e.g. inequality constraints or equality constraints with existentially quantified parameters. In this paper, we focus on existentially quantified distance constraints where the variables are the coordinates of a point $x \in \mathbb{R}^{n}$. As parameters, we have the coordinates of another point $a \in \mathbb{R}^{n}$ and a distance $r \in \mathbb{R}$. Then, the distance constraint fixes the distance between $a$ and $x$ to be equal to $r$. The approximation of such constraints can be useful in many contexts, e.g. GPS localization or parallel robots modeling ([5]).

Permission to make digital or hard copies of all or part of this work for personal or classroom use is granted without fee provided that copies are not made or distributed for profit or commercial advantage and that copies bear this notice and the full citation on the first page. To copy otherwise, to republish, to post on servers or to redistribute to lists, requires prior specific permission and/or a fee.

SAC'O6 April 23-27, 2006, Dijon, France

Copyright 2006 ACM $\ldots \$ 5.00$.
Interval constraint propagation $([2])$ is a widely used technique. It reduces the domains of variables without losing any solution. When it is coupled with some bisection algorithm, an accurate reliable outer approximation of the solution set of the problem is possible. However, when the solution set has a non-null (hyper)volume, this technique will bisect again and again the boxes included inside the solution set, leading to inefficient computations. This situation can be strongly improved using a test for detecting inner boxes. These boxes, which are proved to lie inside the solution set are not bisected any more. Furthermore, such inner boxes often have interesting interpretations in addition to the speedup of computations.

In this paper we propose and compare two different methods for checking if a box is included inside the solution set of a distance equation with existentially quantified parameters. On one hand, using geometric arguments, the quantified distance constraint is changed to an equivalent non quantified disjunction/conjunction of constraints which can be checked using interval arithmetic. On the other hand, the Kaucher arithmetic of generalized intervals ([1]), which represents a new formulation of the modal intervals theory ([6]), allows us to verify the inclusion through a generalized interval evaluation of the constraint. These two tests for inner boxes are implemented in a branch and prune algorithm and experiments have been carried out on academic examples in order to compare these tests.

\section{PROBLEM STATEMENT}

The Euclidean distance between the points $a \in \mathbb{R}^{n}$ and $x \in \mathbb{R}^{n}$ is defined by $f(a, x)=\sqrt{\sum_{k=1}^{n}\left(x_{k}-a_{k}\right)^{2}}$. Given two $n$-dimensional boxes $\mathbf{x}$ and $\mathbf{a}$ and an interval $\mathbf{r}$, we are interested in the following quantified distance constraint

$$
(\exists a \in \mathbf{a})(\exists r \in \mathbf{r})(f(a, x)=r)
$$

which is denoted by $c_{\mathbf{a}, \mathbf{r}}(x)$. The set of $x \in \mathbb{R}^{n}$ which satisfies (1) is denoted by $\rho_{\mathbf{a}, \mathbf{r}}$. This paper aims to provide some sufficient conditions for the inclusion $\mathbf{x} \subseteq \rho_{\mathbf{a}, \mathbf{r}}$.

It can be noted that a sufficient condition designed for one quantified distance constraint can also be used for a conjunction of quantified distance constraints $\bigwedge_{k \in[1 . . m]} c_{\mathbf{a}^{(k)}, \mathbf{r}^{(k)}}(x)$, where $\mathbf{a}^{(k)}$ are some $n$-dimensional boxes and $\mathbf{r}^{(k)}$ some intervals. Indeed, if existentially quantified parameters are not shared between different constraints, we have the following implication:

$$
\bigwedge_{k \in[1 . . m]} \mathbf{x} \subseteq \rho_{\mathbf{a}^{(k)}, \mathbf{r}^{(k)}} \quad \Longrightarrow \quad \mathbf{x} \subseteq \bigcap_{k \in[1 . . m]} \rho_{\mathbf{a}^{(k)}, \mathbf{r}^{(k)}}
$$




\section{A SPECIFIC QUANTIFIER ELIMINATION}

The quantifier elimination (QE) consists in transforming a quantified constraint into an equivalent non quantified constraint. A general algorithm of $\mathrm{QE}$ for polynomial constraints is available ([4]). However, its high complexity restricts its application to very small problems. We propose a specific QE for the distance constraint $c_{\mathbf{a}, \mathbf{r}}(x)$ in the two dimensional case. The three dimensional case can be treated in the same way. However, higher dimensions are still out of the scope of the proposed specific QE, because of the complexity of these problems.

The typical graph of the constraint $c_{\mathbf{a}, \mathbf{r}}(x)$ is shown in Figure 1a. We do not have a direct way to test the inclusion of the box $\mathbf{x}$ in this graph as it is expressed by a quantified constraint. However, it can be reconstructed using the graphs plotted in Figure 1b and Figure 1c. The former consists of eight circles obtained considering the bounds of the intervals a and $\mathbf{r}$. The latter is also simply obtained thanks to these intervals.

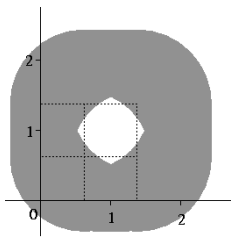

(a)

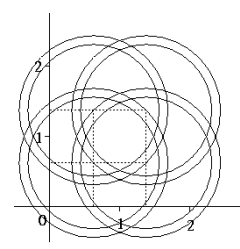

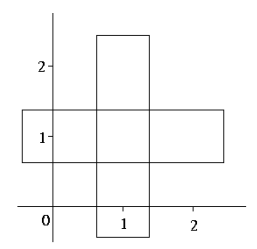

(c)
Figure 1: Typical quantified distance constraint.

Let us call $c_{\mathbf{a}, \mathbf{r}}^{\prime}(x)$ the constraint whose graph is the union of the four exterior disks of Figure $1 \mathrm{~b}$ and the two boxes of Figure 1c. This constraint is expressed by using some simple inequalities. Also, let us call $c_{\mathbf{a}, \mathbf{r}}^{\prime \prime}(x)$ the constraint whose graph is the intersection of the four interior disks of Figure 1b. This constraint is also expressed using some simple inequalities.

It is easily seen that $c_{\mathbf{a}, \mathbf{r}}^{\prime}(x) \wedge \neg c_{\mathbf{a}, \mathbf{r}}^{\prime \prime}(x)$ implies $c_{\mathbf{a}, \mathbf{r}}(x)$. The corresponding real inequalities can be easily checked for a box $\mathbf{x}$ using the interval arithmetic. We eventually obtain a computable sufficient condition for the inclusion $\mathbf{x} \subseteq \rho_{\mathbf{a}, \mathbf{r}}$. Notice that it is not a necessary condition, because a box can satisfy $\mathbf{x} \subseteq \rho_{\mathbf{a}, \mathbf{r}}$ while it does not satisfy the constraint $c_{\mathbf{a}, \mathbf{r}}^{\prime}(x)$. However, it can be proved that given a box satisfying $\mathbf{x} \subseteq \rho_{\mathbf{a}, \mathbf{r}}$, the proposed sufficient condition will prove this inclusion after a finite number of midpoint bisections.

\section{GENERALIZED INTERVAL EVALUATION}

In this section a sufficient condition based on one evaluation of the expression of $f(x, a)$ using generalized intervals and their arithmetic is proposed. This technique was initially proposed in the modal intervals theory $([3,6])$ and is now informally presented in a revisited way using generalized intervals ([1]).

Generalized intervals are defined by relaxing the constraint that bounds have to be ordered, e.g. $[-1,1]$ is a proper interval and $[1,-1]$ is an improper interval. So, related to a set of reals $\{x \in \mathbb{R} \mid u \leq x \leq v\}$, where $u, v \in \mathbb{R}$, one can consider two generalized intervals $[u, v]$ and $[v, u]$. It will be convenient to use the operations dual $[u, v]=[v, u]$ and pro $[u, v]=[\min \{u, v\}, \max \{u, v\}]$ to change the proper/improper quality of a generalized interval keeping unchanged the underlying set of reals. An inclusion is defined for generalized intervals by $\mathbf{x} \subseteq \mathbf{y} \Longleftrightarrow \underline{\mathbf{y}} \leq \underline{\mathbf{x}} \wedge \overline{\mathbf{x}} \leq \overline{\mathbf{y}}$, e.g. $[1,-1] \subseteq[0,1]$.

The Kaucher arithmetic extends the classical intervals arithmetic to generalized intervals and allows the evaluation of an expression with both proper and improper intervals. Based on Kaucher arithmetic, an evaluation of $f($ dual $\mathbf{a}, \mathbf{x}$ ) is calculated. It can be proved that if $\mathbf{r}$ satisfies the inclusion $f($ dual $\mathbf{a}, \mathbf{x}) \subseteq \mathbf{r}$ then

$$
(\forall x \in \mathbf{x})(\exists a \in \mathbf{a})(\exists r \in \mathbf{r})(f(a, x)=r)
$$

is true. The inclusion $f$ (dual $\mathbf{a}, \mathbf{x}) \subseteq \mathbf{r}$ is therefore a sufficient condition for $\mathbf{x} \subseteq \rho_{\mathbf{a}, \mathbf{r}}$. This condition is not necessary in general, e.g. $\mathbf{a}=([-2,2],[-2,2])$ and $\mathbf{r}=[1,1]$ so that $\mathbf{x}=([-2,2],[-2,2])$ is an inner box which does not satisfy $f$ (dual $\mathbf{a}, \mathbf{x}) \subseteq \mathbf{r}$ (in this case, the specific QE presented in Section 3 succeeds in proving the inclusion). However, it can be proved that this sufficient condition is furthermore necessary provided that $\mathbf{x} \cap \mathbf{a}=\emptyset$, which is likely to be met for inner boxes $\mathbf{x}$ in some realistic situations.

\section{CONCLUSION}

Some experiments have been conducted on academic examples of conjunctions of quantified distance constraints. Although both methods are very different, they raise very similar results about both computation times and description of the solution set (with a slight advantage for the test based on generalized intervals). Moreover, the test based on generalized interval evaluation presents two advantages: on one hand, it is much simpler to implement. On the other hand, it can be trivially extended to quantified distance constraints in arbitrary dimensions, where the proposed specific quantifier elimination fails.

As forthcoming work, a new inner test combining the two presented tests will be studied aiming to obtain an optimal test in all situations. Also, we will apply this method in the context of parallel robots study, taking into account the uncertainties on the geometric parameters.

Acknowledgment: We would like to thank Bertrand Neveu for his valuable help during the execution of this work.

\section{REFERENCES}

[1] Goldsztejn A. Définition et Applications des Extensions des Fonctions Réelles aux Intervalles Généralisés. PhD thesis, Université de Nice, Novembre 2005.

[2] Frédéric Benhamou and William J. Older. Applying Interval Arithmetic to Real, Integer and Boolean Constraints. Journal of Logic Programming, 32(1):1-24, 1997.

[3] SIGLA/X group. Modal intervals (basic tutorial). Applications of Interval Analysis to Systems and Control (Proceedings of MISC'99), pages 157-227, 1999.

[4] Davenport J. and Heintz J. Real quantifier elimination is doubly exponential. J. Symb. Comput., 5:29-35, 1988.

[5] Merlet J.P. Parallel robots. Kluwer, Dordrecht, 2000.

[6] Herrero P., M.A. Sainz, Vehí J., and Jaulin L. Quantified set inversion with applications to control. In IEEE International Symposium on Computer Aided Control Systems Design, 2004. 